\title{
Síndrome de Eagle. Una causa poco frecuente de cervicalgia
}

\author{
M. Gelabert-González y A. García-Allut
}

Servicio de Neurocirugía. Hospital Clínico Universitario. Departamento de Cirugía. Universidad de Santiago de Compostela.

\section{Resumen}

El síndrome de Eagle se caracteriza clínicamente por la presencia de dolor facial y/o orofaríngeo en pacientes amigdalectomizados y presentar en los estudios radiológicos un alargamiento de las apófisis estiloides.

Presentamos el caso clínico de una paciente de 49 años con una historia de larga evolución de dolor cervical y en la que los estudios con tomografía computarizada demostraron un alargamiento de ambas apófisis estiloides.

PALABRAS CLAVE: Apófisis estiloides. Neuralgia del glosofaríngeo. Síndrome de Eagle.

\section{Eagle syndrome. An unusual cause of neck pain}

\section{Summary}

Eagle syndrome is characterized by unilateral pain in oropharynx, the side of the face, and cervical region in patients after tonsillectomy. It is caused by an elongated styloid process.

The authors present a case of a woman who suffered from pain in the cervical region during many years. The TC-3D scan showed bilateral elongation of the styloid process.

KEY WORDS: Eagle syndrome. Glossopharyngeal neuralgia. Styloid process.

\section{Introducción}

El síndrome de Eagle fue descrito en el año 1937 por el otorrinolaringólogo alemán W. Eagle en pacientes amigdalectomizados y que presentaban con posterioridad dolor orofaríngeo y cervical y en los que radiográficamente se visualizaba un alargamiento de las apófisis estiloi-

Recibido: 4-05-07. Aceptado: 16-07-07 des $^{1}$. Se trata de una entidad poco conocida en el mundo neuroquirúrgico pero que debe tenerse en consideración en el diagnostico diferencial de algunas cervicalgias y en los casos de neuralgia del glosofaríngeo ${ }^{4}$.

\section{Caso clínico}

Mujer de 49 años que consulta por presentar inestabilidad para la marcha y cervicalgia continua desde hace más de 10 años. El dolor estaba localizado sobre la cara lateral derecha del cuello y se irradiaba hacia el oído y ángulo mandibular del mismo lado. Con ligero incremento del dolor con los movimientos del cuello. Fue amigdalectomizada en la infancia y se realizó septoplastia hace 4 años. Hace 16 años sufrió accidente de circulación con elongación de la columna cervical. En la exploración clínica destacaba un Romberg con mínima lateropulsión derecha y dolor a la presión en caras laterales de la faringe. La radiología simple y tomografía computarizada de la columna cervical demostraron únicamente cambios artrósicos en los espacios $\mathrm{C}_{5}-\mathrm{C}_{6} \mathrm{y} \mathrm{C}_{6}-\mathrm{C}_{7}$. Una TC craneal fue normal.

Se realizó una TC3D del cuello en el que se evidenció un agrandamiento de ambas apófisis estiloides, midiendo la derecha $55 \mathrm{~mm}$ y la izquierda $35 \mathrm{~mm}$, ambas con un defecto de aspecto pseudoartrósico (Figura 1). La paciente rechazó el tratamiento quirúrgico y sigue tratamiento con analgésicos y antinflamatorios.

\section{Discusión}

El síndrome de Eagle se caracteriza por la presencia de dolor en el área orofaríngea y cara debido a un alargamiento y/o calcificación del ligamento estilo-hioideo. Fue descrito en 1937 por el otorrinolaringólogo alemán Eagle en pacientes que habían sido sometidos previamente a una amigdalectomía ${ }^{1}$. Es un síndrome conocido para otorrinolaringólogos y cirujanos maxilofaciales, existiendo pocas referencias tanto en la literatura de habla hispana como en la neuroquirúrgica ${ }^{3,7}$. Aunque aproximadamente un $4 \%$ de la población tiene un alargamiento de las apófisis estiloides, únicamente entre el $4-10.3 \%$ de estos 


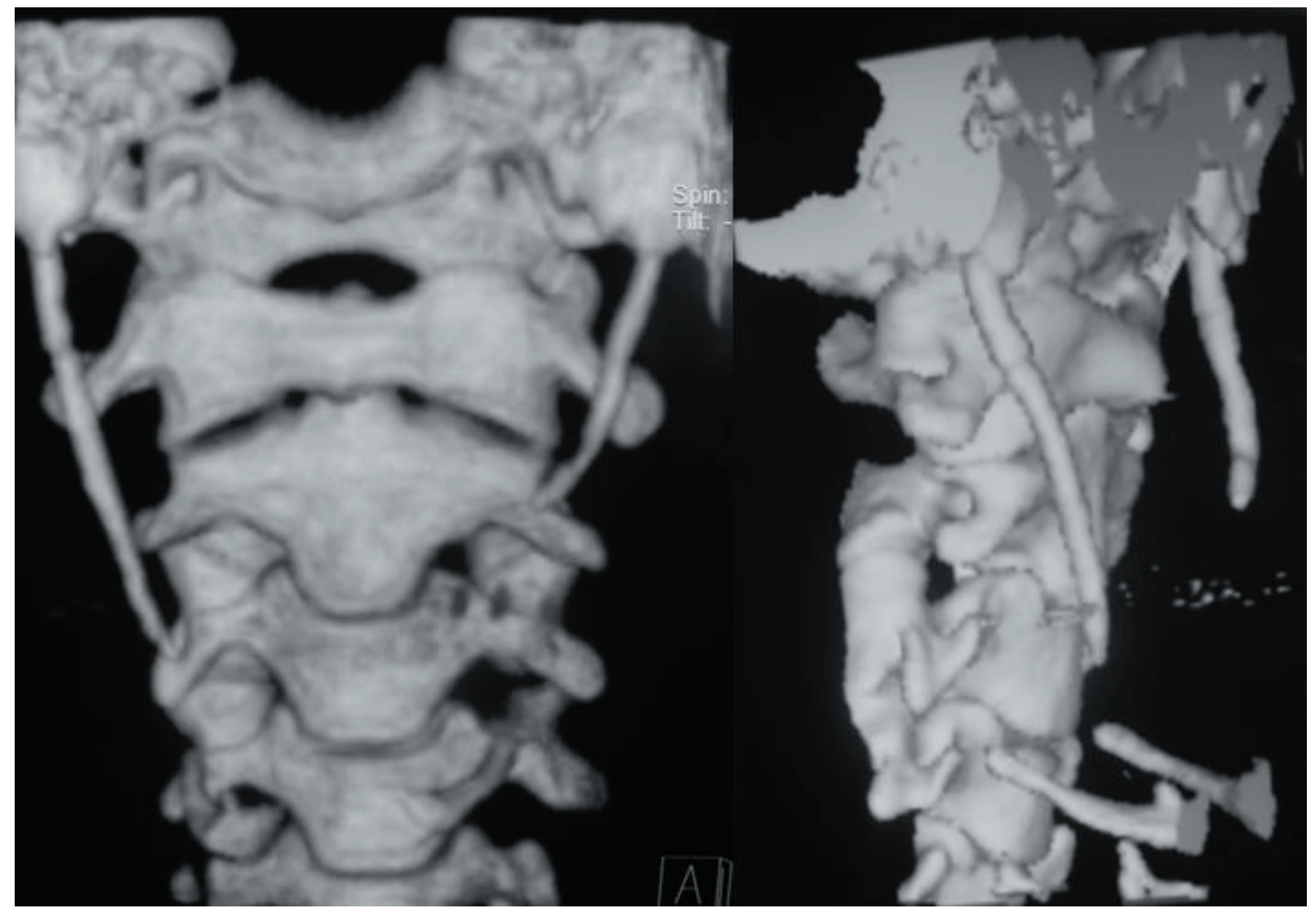

Figura 1. TC con reconstrucción 3D que muestra un agrandamiento de ambas apófisis estiloides, midiendo la derecha $55 \mathrm{~mm}$ y la izquierda $35 \mathrm{~mm}$.

pacientes van a presentar sintomatología relacionada con el síndrome de Eagle 5 .

Clínicamente puede presentarse de dos maneras diferentes. La primera se caracteriza por dolor espástico y persistente en la faringe, en pacientes que pueden haber sido sometidos a amigdalectomía y que tienen unas estiloides mayores de $3 \mathrm{~cm}$ u osificación del ligamento estilo-hioideo. El otro tipo se caracteriza por dolor faríngeo que se acentúa con los movimientos de la cabeza, dolor de cabeza y vértigo en pacientes que no han sido sometidos a cirugías previas ${ }^{8}$. Ambos casos se confunden habitualmente con neuralgia del glosofarínge $\mathrm{o}^{4,6,8}$. Nuestra paciente no se puede incluir de forma clara en ninguno de los dos tipos, aunque estaba sometida a cirugía amigdalar previa, las características del dolor eran similares a las descritas en el segundo tipo, aunque presentaba unas estiloides mayores de $30 \mathrm{~mm}$.

Existen numerosas teorías para explicar la patogenia de este síndrome; la existencia de una hiperplasia o una metaplasia reactiva asociada a una osificación del tejido fibrocartilaginoso del ligamento estilo-hioideo, casi siempre como respuesta a una traumatismo previo. Este fenómeno explicaría la aparición del síndrome en pacientes sometidos previamente a una amigdalectomía, como había descrito inicialmente W. Eagle ${ }^{1}$. Para otros el dolor es causado por una compresión del nervio glosofaríngeo a su paso por la apófisis estiloides elongada. El complejo estilo-hioideo está formado por la apófisis estiloides, el ligamento estilo-hioideo y el cuerno del hueso hioides que derivan del segundo arco branquial. En ocasiones se puede observar calcificaciones segmentarias de este complejo en pacientes asintomáticos. Esta teoría podría explicar la aparición del síndrome en pacientes que no fueron sometidos a amigdalectomía o que sufrieran traumatismos previos al desarrollo del cuadro clínico ${ }^{2}$. Para algunos autores, esta situación debería denominarse síndrome estilohiodeo para diferenciarlo del verdadero Eagle en el cual debe haber amigdalectomía previa ${ }^{11}$. Una cuarta teoría habla de que la sintomatología es una consecuencia del envejecimiento, ya que debido a la pérdida de elasticidad de los tejidos, es más frecuente la existencia de tendinitis o reacciones inflamatorias que causan dolor en el territorio glosofaríngeo simulando un síndrome de Eagle. Para evitar confusiones se ha denominado a esta situación como síndrome pseudoestilo-hioideo 9 .

El diagnóstico se basa fundamentalmente en la clínica y los estudios radiográficos. En la exploración física se puede palpar la estiloides dentro de la fosa tonsilar, desencadenando dolor intenso. La radiología simple de la columna cervical muestra el alargamiento de la estiloides que se confirma con tomografía computarizada al observar una estiloides, que en condiciones normales mide hasta $2.5 \mathrm{~cm}$., alcanza en estos casos una longitud superior a $\operatorname{los} 3 \mathrm{~cm}$. 
El tratamiento es inicialmente farmacológico incluyendo infiltraciones locales con esteroides y anestésicos locales $^{5,10}$. Cuando falla el tratamiento médico esta indicada la resección quirúrgica siguiendo una vía orofaríngea como describió inicialmente Eagle ${ }^{1}$ y defendida por otros autores ${ }^{3,7}$ o bien usando una vía lateral en el cuello ${ }^{5}$.

\section{Bibliografía}

1. Eagle, W.W.: Elongated styloid processes: report of two cases. Arch Otolaryngol 1937; 25: 584-587.

2. Graf, C.J.: Glossopharyngeal neuralgia and ossification of the stylohyoid ligament. J Neurosurg 1958; 16: 448-453.

3. Miyar, V., Morais, D., Santos, J.: Cirugía del síndrome de la estiloides alargada. An Otorrinolaringol Ibero AM 1997; 24: 303-309.

4. Molet, J., Chong, C., Quintanilla, M., et al.: Microdescompresión vascular en neuralgias del glosofaríngeo. Neurocirugía 1995; 6: 227-229.

5. Murtagh, R.D., Caracciolo, J.T., Fernandez, G.: CT findings associated with eagle syndrome. AJNR 2001; 22: 14011402.

6. Renzi, G., Mastellone, P., Leonardi, A., Becelli, R., Bonamini, M., Fini, G.: Basicranium malformation with anterior dislocation of right styloid process causing stylalgia. $\mathrm{J}$
Craniofac Surg. 2005;16: 418-420.

7. Roca, A., Armengot, M., Giménez, G., Barona, R., Basterra, J.: Tratamiento quirúrgico del síndrome de Eagle por vía orofaríngea. Presentación de un caso. Acta Otorrinolaringol Esp 1992; 43: 210-212.

8. Slavin, K.V.: Eagle syndrome: entrapment of the glossopharyngeal nerve? Case report and review of the literature. J Neurosurg 2002; 97: 216-218.

9. Steimann, E.P.: Styloid syndrome in absence of an elongated process. Acta Otolaryngol 1968; 66: 347-356.

10. Sun, C.K., Mercuri, V., Cook, M.J.: Eagle syndrome. An unusual cause of head and neck pain. Arch Neurol 2006; 63: 294-295

11. van der Westhuijzen, A.J., van der Merwe, J., Grotespass, F.W.: Eagle's syndrome: lesser cornu amputation: an alternative surgical solution? Int J Oral Maxillofac Surg 1999; 28:335-337.

Gelabert-González, M.; García-Allut, A.: Síndrome de Eagle. Una causa poco frecuente de cervicalgia. Neurocirugía 2008; 19: 254-256.

Correspondencia postal: M. Gelabert González. Servicio de Neurocirugía. Hospital Clínico Universitario. La Choupana s/n. 15706 Santiago de Compostela. 